\title{
Influência da qualidade micotoxicológica e nutricional de híbridos de milho no custo da ração de frangos de corte
}

\author{
[Influence of mycotoxicological and nutritional quality of maize hybrids on broiler chicken \\ feed cost] \\ A.O. Mallmann ${ }^{1}$, P. Dilkin ${ }^{1}$, J.K. Vidal ${ }^{2}$, G.R. Meinerz ${ }^{3}$, \\ M.S. Oliveira ${ }^{1}$, C.A. Mallmann ${ }^{1}$ \\ ${ }^{1}$ Centro de Ciências Rurais - Universidade Federal de Santa Maria - Santa Maria, RS \\ ${ }^{2}$ Aluno de pós-graduação - Centro de Ciências Rurais - Universidade Federal \\ de Santa Maria - Santa Maria, RS \\ ${ }^{3}$ Universidade Federal da Fronteira Sul - Cerro Largo, RS
}

\begin{abstract}
RESUMO
Objetivou-se avaliar as variáveis micotoxicológicas e nutricionais de híbridos de milho com diferentes características que influenciam no custo da ração para frangos de corte. Foram avaliados 26 híbridos de milho geneticamente modificados nas safrinhas de 2016 e 2017, com diferentes germoplasmas, textura de endosperma e duração do ciclo. Nos híbridos, foram avaliados grãos avariados, fumonisinas $\left(\mathrm{B}_{1}+\mathrm{B}_{2}\right)$ (FUM), aflatoxinas $\left(B_{1}+B_{2}+G_{1}+G_{2}\right)$ (AFLA), zearalenona (ZEA), deoxinivalenol (DON), umidade, proteína bruta $(\mathrm{PB})$, energia metabolizável aparente corrigida para balanço de nitrogênio (EMAn), aminoácidos digestíveis para aves (lisina, metionina, cistina e treonina) e o respectivo custo da ração inicial para frangos de corte, que foi calculada pelo custo mínimo. A prevalência de FUM, AFLA, ZEA e DON foi de 90, 17, 33 e 0\%, com médias de 3067, 1, 38 e $0 \mu \mathrm{g} / \mathrm{kg}$ nos dois anos, respectivamente. A média de EMAn e PB foi de $3264 \mathrm{kcal} / \mathrm{kg}$ e $8,02 \%$, respectivamente, e diferiu $(\mathrm{P}<0,05)$ nos dois anos. $\mathrm{O}$ custo da ração foi influenciado significativamente $(\mathrm{P}<0,05)$ por FUM, PB, EMAn nos dois anos. Híbridos com tecnologia Viptera apresentam menor concentração por FUM e menor custo da ração. Híbridos de ciclo precoce têm menor concentração de FUM, maiores percentuais de PB e de aminoácidos digestíveis e menor custo da ração.
\end{abstract}

Palavras-chave: aflatoxinas, fumonisinas, zearalenona, energia, proteína bruta

\begin{abstract}
The objective of this study was to evaluate the mycotoxicological and nutritional variables of maize hybrids with different characteristics that influence the broiler chicken's feed costs. In 2016 and 2017 winter crops, 26 genetically modified hybrids of maize with different germplasm, endosperm texture and cycle duration were evaluated. The analyzed variables were damaged grains, fumonisins $\left(B_{1}+B_{2}\right)(F U M)$, aflatoxins $\left(B_{1}+B_{2}+G_{1}+G_{2}\right)$ (AFLA), zearalenone ( $\left.Z E A\right)$, deoxynivalenol (DON), moisture, crude protein $(C P)$, apparent metabolizable energy corrected for nitrogen balance (AMEn), digestible amino acids for poultry (lysine, methionine, cystine and threonine) and the respective cost of the initial feed for broiler chickens calculated at the minimum cost. The prevalence of FUM, AFLA, ZEA and DON was 90, 17, 33 and $0 \%$, with means of $3067,1,38$ and $0 \mu \mathrm{g} / \mathrm{kg}$ in the two years, respectively. The mean of AMEn and CP was $3264 \mathrm{kcal} / \mathrm{kg}$ and $8.02 \%$, respectively, and differed $(P<0.05)$ in the two years. The feed cost was significantly influenced $(P<0.05)$ by FUM, PB, AMEn in two years. Hybrids with Viptera technology show lower concentration per FUM and lower feed cost. Early cycle hybrids have lower concentrations of FUM, higher percentages of CP and digestible amino acids, and lower feed costs.
\end{abstract}

Keywords: aflatoxins, fumonisins, zearalenone, energy, crude protein

Recebido em 16 de março de 2018

Aceito em 18 de dezembro de 2018

E-mail: aomallmann@gmail.com 


\section{INTRODUÇÃO}

O milho (Zea mays L.) é o cereal mais produzido no mundo, com produção projetada em 1.044 milhões de toneladas na safra de 2017/2018. Essa produção concentra-se principalmente nos EUA, na China e no Brasil, com 370, 216 e 95 milhões de toneladas colhidas, respectivamente (World..., 2017). No Brasil, estimativas indicam que 41,5 milhões de toneladas foram utilizadas para fabricação de rações para animais no ano de 2017 (Efeitos..., 2017), demonstrando que o milho é o cereal mais utilizado como ingrediente de dietas para animais.

O crescimento fúngico e a produção de micotoxinas no milho são influenciados por diversos fatores bióticos e abióticos (Dilkin et al., 2002). O milho brasileiro está frequentemente contaminado com diversas micotoxinas, como aflatoxinas $\left(\mathrm{B}_{1}, \mathrm{~B}_{2}, \mathrm{G}_{1}\right.$ e $\left.\mathrm{G}_{2}\right)$, fumonisinas $\left(\mathrm{B}_{1} \quad\right.$ e $\left.\quad \mathrm{B}_{2}\right)$, zearalenona, deoxinivalenol, entre outras (Oliveira et al., 2017). As micotoxinas afetam os animais de forma individual ou sinérgica na presença de mais de uma micotoxina, podendo afetar vários órgãos, como o trato gastrointestinal, o fígado e o sistema imunológico, resultando essencialmente na redução da produtividade das aves e na mortalidade em casos extremos (Murugesan et al., 2015).

Nos últimos anos, houve um vasto desenvolvimento de híbridos de milho geneticamente modificados, aos quais foram inseridos genes de Bacillus thuringiensis, que levam à produção de proteínas tóxicas a algumas ordens de insetos consideradas pragas da cultura do milho. Plantas de milho de híbridos com essa tecnologia sofrem menor ataque de insetos, fazendo com que a planta, teoricamente, seja menos vulnerável aos fungos produtores de micotoxinas (Hammond et al., 2004). Em estudo realizado com 36 cultivares de milho, Pinto et al. (2007) relataram também efeito de diferentes híbridos na incidência de grãos ardidos e mofados, bem como acentuada interação entre as cultivares e o fungo toxigênico Fusarium subglutinans quanto à biossíntese de fumonisina $\mathrm{B}_{1}$ em grãos de milho. Assim, fica evidenciado que a variabilidade genética dos híbridos de milho e sua relação com a contaminação por micotoxinas é um fator relevante na escolha do material genético mais adequado para cultivo.
Não obstante o aspecto micotoxicológico, o milho é o mais importante componente energético das rações (Moreira et al., 1994), mas pode apresentar variações na sua composição nutricional, ocasionadas por fatores como potencial genético, nível de adubação utilizado, fertilidade do solo e condições climáticas (Eyng et al., 2009). Nesse sentido, a informação mais exata e precisa da matriz nutricional dos alimentos é essencial para a formulação de rações com custo mínimo e redução dos riscos de suprimento de níveis marginais ou em excesso, com a finalidade de otimizar a produtividade e maximizar a rentabilidade na produção animal (Silva et al., 2008).

Como consequência desses fatores, a indústria precisa exigir maior qualidade de grãos para evitar a necessidade de uso exagerado de aditivos antimicotoxinas para minimizar os efeitos tóxicos das micotoxinas bem como de óleos e aminoácidos sintéticos para suprir as exigências nutricionais. Considerando que a recomendação de híbridos de milho que atendam a esses requisitos ainda carece de pesquisas, o objetivo deste trabalho foi avaliar as variáveis micotoxicológicas e nutricionais de híbridos de milho com diferentes características que influenciam no custo da ração para frangos de corte.

\section{MATERIAL E MÉTODOS}

Empregaram-se 26 híbridos de milho geneticamente modificados, em cultivos extemporâneos (safrinha) (fevereiro a junho) dos anos de 2016 e 2017, em uma área experimental localizada no extremo oeste do estado do Paraná, Brasil (Tab. 1). O delineamento experimental utilizado foi de blocos ao acaso, com quatro repetições. As parcelas foram constituídas de seis fileiras de $5 \mathrm{~m}$ de comprimento, com espaçamento de $0,45 \mathrm{~m}$ e avaliação das quatro fileiras centrais. Após a colheita, foi coletado $1,25 \mathrm{~kg}$ de grãos de cada parcela, dos quais $250 \mathrm{~g}$ foram separados visualmente em grãos aparentemente sadios e grãos avariados (Brasil, 2011). As amostras de $1 \mathrm{~kg}$ foram parcialmente secas em estufa de ar forçado a $55^{\circ} \mathrm{C}$, por $48 \mathrm{~h}$, moídas empregando-se peneira de $1 \mathrm{~mm}$ e homogeneizadas. 
Influência da qualidade...

Tabela 1. Caracterização dos híbridos de milho cultivados nas safrinhas de 2016 e 2017

\begin{tabular}{|c|c|c|c|c|c|c|c|c|c|c|c|}
\hline Híbrido & Transgenia & $\begin{array}{l}\text { Tipo de } \\
\text { híbrido }^{1}\end{array}$ & $\mathrm{Ciclo}^{2}$ & Cor $^{3}$ & Textura $^{4}$ & Híbrido & Transgenia & $\begin{array}{l}\text { Tipo de } \\
\text { híbrido }\end{array}$ & Ciclo & Cor & Textura \\
\hline 1 & PW & HT & SP & $\mathrm{AM} / \mathrm{AL}$ & SMDU & 14 & PW & HS & $\mathrm{P}$ & AM & SMDE \\
\hline 2 & PW & HT & SP & $\mathrm{AM} / \mathrm{AL}$ & SMDU & 15 & PW & HT & $\mathrm{P}$ & $\mathrm{AL}$ & SMDU \\
\hline 3 & PW & HT & $\mathrm{P}$ & $\mathrm{AL}$ & SMDU & 16 & VIPTERA & $\mathrm{HSm}$ & $\mathrm{P}$ & AM/AL & DURO \\
\hline 4 & PW & HS & $\mathrm{P}$ & $\mathrm{AM} / \mathrm{AL}$ & SMDE & 17 & PRO2 & HS & SP & AM/AL & SMDE \\
\hline 5 & PW & HS & $\mathrm{P}$ & $\mathrm{AM} / \mathrm{AL}$ & SMDE & 18 & PRO3 & HS & SP & AM/AL & DENT \\
\hline 6 & PW & HT & $\mathrm{P}$ & $\mathrm{AM} / \mathrm{AL}$ & SMDU & 19 & VIPTERA & HD & $\mathrm{P}$ & $\mathrm{AL}$ & DURO \\
\hline 7 & PW & HS & $\mathrm{N}$ & $\mathrm{AL}$ & SMDU & 20 & VIPTERA & HS & SP & $\mathrm{AL}$ & DURO \\
\hline 8 & PRO3 & HS & $\mathrm{P}$ & $\mathrm{AM} / \mathrm{AL}$ & SMDE & 21 & PW & HS & $\mathrm{P}$ & AM/AL & SMDU \\
\hline 9 & PRO3 & HS & SP & AM & SMDE & 22 & PW & HSm & $\mathrm{P}$ & AM/AL & SMDU \\
\hline 10 & PRO3 & HS & SP & AL & SMDU & 23 & PRO & HS & $\mathrm{P}$ & LR & SMDU \\
\hline 11 & PRO3 & HS & SP & AL & SMDU & 24 & HX & HS & SP & AL & SMDU \\
\hline 12 & PW & HT & SP & $\mathrm{AM} / \mathrm{AL}$ & SMDU & 25 & HX & HS & SP & AV & DURO \\
\hline 13 & PW & HT & $\mathrm{P}$ & $\mathrm{AM} / \mathrm{AL}$ & SMDE & 26 & VIPTERA & HS & $\mathrm{P}$ & $\mathrm{AL}$ & DURO \\
\hline
\end{tabular}

${ }^{1}$ Tipo de híbrido: HS - híbrido simples; HSm - híbrido simples modificado; HD - híbrido duplo; HT - híbrido triplo. ${ }^{2}$ Ciclo: SP - superprecoce; $\mathrm{P}$ - precoce; $\mathrm{N}$ - normal. ${ }^{3}$ Cor do Grão: AL - alaranjado; LR - laranja; AV avermelhado; AM - amarelo. ${ }^{4}$ Textura de endosperma: DENT - dentado; SMDE - semidentado; SMDU - semiduro.

Uma fração da amostra foi submetida à extração das aflatoxinas $\left(\mathrm{B}_{1}+\mathrm{B}_{2}+\mathrm{G}_{1}+\mathrm{G}_{2}\right)$ (AFLA), das fumonisinas $\left(\mathrm{B}_{1}+\mathrm{B}_{2}\right)$ (FUM), da zearalenona (ZEA) e do deoxinivalenol (DON) em solução de ACN e $\mathrm{H}_{2} \mathrm{O}$, agitada por $20 \mathrm{~min}$ a $2.500 \mathrm{rpm}$ em multivórtex. Uma alíquota do extrato foi injetada em cromatógrafo líquido de alta eficiência 1200 Series Infinity (Agilent, EUA), acoplado a um espectrômetro de massas (LC-MS/MS) 5500 QTRAP (Applied Biosystems, EUA). As análises foram efetuadas seguindo-se os parâmetros propostos por Sulyok et al. (2007), Mallmann et al. (2013) e Berthiller et al. (2005), para AFLA, FUM e ZEA/DON, respectivamente.

A determinação dos valores de energia metabolizável aparente para aves corrigida para balanço de nitrogênio (EMAn), proteína bruta (PB), umidade e os aminoácidos lisina (LisD), metionina (MetD), cistina (CisD) e treonina (TreD) digestíveis para aves foi realizada pelo método de espectroscopia no infravermelho próximo (NIRS), efetuada pelo Laboratório CEAN Adisseo Brasil e Adisseo France S.A.S. (PNE, 2017). O espectrômetro NIRS XDS (Foss, Dinamarca) utilizado foi calibrado com equações de predição baseadas em análises de aminoácidos, ácido fítico e conteúdo total de fósforo por cromatografia líquida de alta eficiência e em métodos in vivo para determinar a digestibilidade de aminoácidos e EMAn.

Todas as variáveis foram ajustadas para $87 \%$ de matéria seca para o cálculo do custo de rações para frangos de corte na fase inicial (do $10^{-}$ao $21^{\text {o }}$ dia de idade) ( $\mathrm{R} \$ / \mathrm{t}$ ração) utilizando-se a planilha PPFR (Unesp, Brasil) de formulação não linear (ração de custo mínimo). As exigências nutricionais seguiram as recomendações de Rostagno et al. (2011); os padrões da agroindústria e os custos dos insumos foram os praticados no mercado regional do oeste do estado do Paraná, em julho de 2017 (Tab. 2). A dose de inclusão de aditivo antimicotoxinas (AAM) foi calculada proporcionalmente ao nível de concentração micotoxicológica de cada híbrido após o ajuste das FUM para fumonisinas totais, que são a soma das fumonisinas livres (FUM) e das fumonisinas ocultas, conforme a equação proposta por Oliveira et al. (2015): fumonisinas totais $(\mathrm{mg} / \mathrm{kg})=\left(0,8583+0,5615^{*} \mathrm{FUM}\right)^{2}$. Em seguida, a dose de AAM foi calculada na proporção de $2,5 \mathrm{~kg}$ de $\mathrm{AAM} / \mathrm{t}$ de ração para cada $10.000 \mu \mathrm{g} / \mathrm{kg}$ de fumonisinas totais, acrescida da proporção de $2,5 \mathrm{~kg}$ de $\mathrm{AAM} / \mathrm{t}$ de ração para cada $28 \mu \mathrm{g} / \mathrm{kg}$ de AFLA.

Todas as variáveis foram submetidas à análise de variância e à verificação do efeito de blocos. Utilizou-se o teste de Scott-Knott (variáveis paramétricas) para agrupar os híbridos com características semelhantes ou o teste de KruskalWallis (variáveis não paramétricas) por ano de cultivo. Utilizou-se o teste-F (variáveis paramétricas) ou o teste de Mann-Whitney (variáveis não paramétricas) para verificação do efeito de ano. As variáveis foram submetidas à análise de regressão simples e à análise de regressão múltipla pelo procedimento Stepwise 
para gerar o modelo de predição do custo da ração. Utilizou-se o nível de significância de 5\% para todas as análises. Realizou-se uma análise dos componentes principais (PCA). Os dados foram analisados no programa estatístico Statgraphics Centurion XV, versão 15.2.11 (Manugistics Inc., Rockville, MD, EUA).

Tabela 2. Exigências nutricionais e custos dos insumos utilizados para o cálculo de custo de rações para frangos de corte na fase inicial (do $10^{\circ}$ ao $21^{\circ}$ dia de idade)

\begin{tabular}{lccc}
\hline \multicolumn{1}{c}{ Exigências nutricionais } & Quantidade & Custo dos ingredientes & $\mathrm{R} \$ / \mathrm{kg}$ \\
\hline Energia metabolizável aves (corr. balanço de N) (kcal/kg) & 3100 & Milho & 0,30 \\
Proteína bruta (\%) & 21,20 & Farelo de soja 46\% & 0,98 \\
Metionina digestível aves (\%) & 0,5541 & Farinha de carne e ossos 45\% & 0,77 \\
Metionina + cistina digestível aves (\%) & 0,8590 & Óleo de soja & 2,27 \\
Lisina digestível aves (\%) & 1,1940 & DL-hidroximetionina 88\% & 6,71 \\
Treonina digestível aves (\%) & 0,7760 & L-lisina HCL 64,1\% & 2,55 \\
Cálcio total (\%) & 0,83 & L-treonina 98,5\% & 6,60 \\
Fósforo disponível (\%) & 0,38 & Calcário calcítico & 0,06 \\
Sódio (\%) & 0,22 & Fosfato bicálcico & 1,30 \\
Premix vitamínico mineral (\%) & 0,20 & Sal comum & 0,33 \\
& & Premix vitamínico mineral & 10,43 \\
& & Aditivo antimicotoxinas & 7,35 \\
\hline
\end{tabular}

\section{RESULTADOS E DISCUSSÃO}

$\mathrm{Na}$ análise dos grãos avariados, apesar de o percentual ter sido maior $(\mathrm{P}<0,05)$ no ano de 2016 em relação a 2017, apenas três híbridos tiveram média superior ao limite de classificação para o milho tipo 1, que é de 6\% (Brasil, 2011). Em 2016, a distribuição dos híbridos foi em três grupos; em 2017, em dois grupos. Os índices médios variaram entre 1,2 e $10,9 \%$ nos dois anos de avaliação, demonstrando que a variabilidade nos percentuais de grãos avariados foi influenciada $(\mathrm{P}<0,05)$ por híbrido e por ano de cultivo (Tab. 3).

Na análise das FUM, a distribuição dos híbridos se deu em três grupos no ano de 2016 e em apenas dois grupos no ano de 2017. As FUM foram detectadas em $90 \%$ das amostras e houve interação $(\mathrm{P}<0,05)$ entre os anos de avaliação, em que 19 dos 26 híbridos avaliados tiveram média de concentração maior $(\mathrm{P}<0,05)$ no ano de 2016 em relação a 2017, o que demonstra a variabilidade das FUM nos híbridos em função do ano (Tab. 3). Prevalência, variabilidade e média de concentração por FUM semelhante foram relatadas por Camargos et al. (2000), que compararam 19 híbridos de milho cultivados em campos experimentais em três locais no estado de São Paulo, Brasil, durante a safra 1997/1998 e constataram que todas as amostras estavam contaminadas com FUM, com média de $7470 \mu \mathrm{g} / \mathrm{kg}(2080-34290 \mu \mathrm{g} / \mathrm{kg})$.

A concentração de FUM nos híbridos, associada à textura dos grãos, não apresentou interação $(\mathrm{P}>0,05)$, característica dos híbridos que vem sendo discutida com relação à susceptibilidade à infestação fúngica (Tab. 4). No entanto, dos cinco híbridos com textura dura, quatro são da tecnologia de transgenia Viptera, os quais tiveram menores $(\mathrm{P}<0,05)$ concentrações de FUM. Quanto mais macia a textura do grão, maior a suscetibilidade a incidências de doenças e infestação por insetos que podem causar injúrias aos grãos e consequentemente favorecer a colonização de fungos (Ono et al., 2006; Buerstmayr et al., 2003; Bútron et al., 2009). Ao avaliarem 20 variedades crioulas de milho da região Sul do Brasil, Oliveira et al. (2009) constataram que quanto maior a proporção de endosperma vítreo, que é mais denso em relação ao farináceo, maior a força necessária até a ruptura do grão e menor a contaminação por Fusarium sp. No entanto, no mesmo estudo, os autores não observaram correlação com a concentração de ZEA, que foi detectada em $75 \%$ das amostras (50-640 $\mu \mathrm{g} / \mathrm{kg})$. Quanto às classificações dos híbridos por ciclo e tipo, híbridos de ciclo precoce tiveram menores $(\mathrm{P}<0,05)$ concentrações de FUM, e não foi observada interação $(\mathrm{P}>0,05)$ com o tipo de híbrido (Tab. 4). 
Influência da qualidade...

Tabela 3. Médias de grãos avariados (AV), fumonisinas $\left(B_{1}+B_{2}\right)(F U M)$, aflatoxinas $\left(B_{1}+B_{2}+G_{1}+G_{2}\right)$ (AFLA), zearalenona (ZEA) e deoxinivalenol (DON) de 26 híbridos de milho cultivados nas safrinhas de 2016 e 2017

\begin{tabular}{|c|c|c|c|c|c|c|c|c|c|c|c|c|c|c|c|}
\hline \multirow{2}{*}{ Híbrido } & \multicolumn{3}{|c|}{ AV (\%) } & \multicolumn{3}{|c|}{ FUM $(\mu \mathrm{g} / \mathrm{kg})$} & \multicolumn{3}{|c|}{ AFLA $(\mu \mathrm{g} / \mathrm{kg})$} & \multicolumn{3}{|c|}{ ZEA $(\mu \mathrm{g} / \mathrm{kg})$} & \multicolumn{3}{|c|}{ DON $(\mu \mathrm{g} / \mathrm{kg})$} \\
\hline & 2016 & 2017 & $\mathrm{P}$ & 2016 & 2017 & $\mathrm{P}$ & 2016 & 2017 & $\mathrm{P}$ & 2016 & 2017 & $\mathrm{P}$ & 2016 & 2017 & $\mathrm{P}$ \\
\hline 1 & $2,48 \mathrm{c}$ & $4,50 \mathrm{a}$ & 0,039 & $14833 a$ & $1198 \mathrm{a}$ & 0,006 & 0 & 4,9 & 0,131 & 5 & $275 a$ & 0,018 & 0 & 0 & - \\
\hline 2 & $2,13 \mathrm{c}$ & $2,33 b$ & 0,794 & $5195 c$ & $1402 \mathrm{a}$ & 0,017 & 0 & 0,0 & - & 0 & $7 b$ & 0,248 & 0 & 0 & - \\
\hline 3 & $1,87 \mathrm{c}$ & $1,93 b$ & 0,816 & $2560 \mathrm{c}$ & $184 b$ & 0,017 & 0 & 0,0 & - & 0 & $142 \mathrm{a}$ & 0,037 & 0 & 0 & - \\
\hline 4 & $4,87 \mathrm{c}$ & $2,33 b$ & 0,079 & $3289 \mathrm{c}$ & $933 a$ & 0,167 & 0 & 0,0 & - & 0 & $55 b$ & 0,121 & 0 & 0 & - \\
\hline 5 & $3,58 \mathrm{c}$ & $3,83 \mathrm{a}$ & 0,879 & $2400 \mathrm{c}$ & $334 b$ & 0,001 & 0 & 3,3 & 0,248 & 0 & $43 b$ & 0,078 & 0 & 0 & - \\
\hline 6 & $4,37 \mathrm{c}$ & $1,33 b$ & 0,073 & $2262 c$ & $333 b$ & 0,031 & 0 & 0,0 & - & 0 & $8 b$ & 0,317 & 0 & 0 & - \\
\hline 7 & $10,9 \mathrm{a}$ & $3,50 \mathrm{a}$ & 0,081 & $8380 \mathrm{~b}$ & $1802 \mathrm{a}$ & 0,004 & 0 & 11,8 & 0,121 & 0 & $266 a$ & 0,037 & 0 & 0 & - \\
\hline 8 & $2,90 \mathrm{c}$ & $3,00 \mathrm{a}$ & 0,374 & $3432 c$ & $1093 a$ & 0,035 & 0 & 0,0 & - & 0 & $0 \mathrm{~b}$ & - & 0 & 0 & - \\
\hline 9 & $7,67 b$ & $2,17 \mathrm{~b}$ & 0,045 & $2318 \mathrm{c}$ & $776 \mathrm{~b}$ & 0,064 & 0 & 0,0 & - & 18 & $0 \mathrm{~b}$ & 0,317 & 0 & 0 & - \\
\hline 10 & $2,93 \mathrm{c}$ & $3,83 \mathrm{a}$ & 0,554 & $9210 b$ & $958 \mathrm{a}$ & 0,026 & 0 & 5,2 & 0,121 & 7 & $354 a$ & 0,246 & 0 & 0 & - \\
\hline 11 & $3,15 \mathrm{c}$ & $1,17 \mathrm{~b}$ & 0,008 & $5840 \mathrm{c}$ & $52 \mathrm{~b}$ & 0,000 & 0 & 0,0 & - & 0 & $28 \mathrm{~b}$ & 0,248 & 0 & 0 & - \\
\hline 12 & $4,70 \mathrm{c}$ & $1,83 b$ & 0,062 & $7383 b$ & $214 b$ & 0,004 & 0 & 0,0 & - & 17 & $40 \mathrm{~b}$ & 0,487 & 0 & 0 & - \\
\hline 13 & $2,58 \mathrm{c}$ & $1,50 \mathrm{~b}$ & 0,422 & $2553 c$ & $612 b$ & 0,045 & 0 & 0,0 & - & 0 & $17 \mathrm{~b}$ & 0,131 & 0 & 0 & - \\
\hline 14 & $3,03 \mathrm{c}$ & $2,67 \mathrm{~b}$ & 0,787 & $6690 \mathrm{c}$ & $902 a$ & 0,247 & 0 & 0,4 & 0,248 & 7 & $86 a$ & 0,067 & 0 & 0 & - \\
\hline 15 & $3,47 \mathrm{c}$ & $2,50 \mathrm{~b}$ & 0,076 & $5406 c$ & $236 b$ & 0,019 & 0 & 22,9 & 0,186 & 0 & $43 b$ & 0,079 & 0 & 0 & _ \\
\hline 16 & $4,00 \mathrm{c}$ & $1,83 b$ & 0,211 & $2542 c$ & $295 b$ & 0,021 & 0 & 0,0 & - & 0 & $58 \mathrm{a}$ & 0,037 & 0 & 0 & - \\
\hline 17 & $5,43 \mathrm{c}$ & $3,67 \mathrm{a}$ & 0,125 & $11798 \mathrm{a}$ & $916 a$ & 0,015 & 0 & 0,0 & - & 10 & $33 b$ & 0,266 & 0 & 0 & - \\
\hline 18 & $3,70 \mathrm{c}$ & $1,83 b$ & 0,437 & $5600 \mathrm{c}$ & $344 b$ & 0,000 & 0 & 2,1 & 0,414 & 0 & $21 b$ & 0,414 & 0 & 0 & - \\
\hline 19 & $4,40 \mathrm{c}$ & $3,33 \mathrm{a}$ & 0,398 & $3448 c$ & $281 b$ & 0,001 & 0 & 0,0 & - & 0 & $164 a$ & 0,037 & 0 & 0 & - \\
\hline 20 & $1,90 \mathrm{c}$ & $2,00 \mathrm{~b}$ & 0,872 & $4525 c$ & $231 b$ & 0,005 & 0 & 0,0 & - & 0 & $56 \mathrm{~b}$ & 0,078 & 0 & 0 & - \\
\hline 21 & $3,23 \mathrm{c}$ & $1,67 b$ & 0,076 & $4405 c$ & $1544 a$ & 0,165 & 0 & 0,0 & - & 0 & $10 \mathrm{~b}$ & 0,317 & 0 & 0 & - \\
\hline 22 & $3,60 \mathrm{c}$ & $2,50 \mathrm{~b}$ & 0,596 & $4044 c$ & $741 b$ & 0,292 & 0 & 0,0 & - & 0 & $26 \mathrm{~b}$ & 0,317 & 0 & 0 & - \\
\hline 23 & $7,37 \mathrm{~b}$ & $2,00 \mathrm{~b}$ & 0,036 & $4024 c$ & $650 \mathrm{~b}$ & 0,146 & 0 & 0,0 & - & 0 & $25 \mathrm{~b}$ & 0,317 & 0 & 0 & - \\
\hline 24 & $1,75 \mathrm{c}$ & $2,00 \mathrm{~b}$ & 0,841 & $5023 c$ & $1402 \mathrm{a}$ & 0,128 & 0 & 0,0 & - & 0 & $54 \mathrm{~b}$ & 0,078 & 0 & 0 & - \\
\hline 25 & $3,77 \mathrm{c}$ & $2,17 \mathrm{~b}$ & 0,038 & $11080 \mathrm{a}$ & $1767 a$ & 0,003 & 0 & 0,9 & 0,317 & 0 & $19 \mathrm{~b}$ & 0,317 & 0 & 0 & - \\
\hline 26 & $3,27 \mathrm{c}$ & $1,63 b$ & 0,247 & $1632 c$ & $443 b$ & 0,003 & 0 & 0,6 & 0,186 & 0 & $83 b$ & 0,186 & 0 & 0 & - \\
\hline $\mathrm{P}$ & 0,001 & 0,002 & & 0,000 & 0,048 & & - & - & & - & 0,040 & & - & - & \\
\hline Ano & 2016 & 2017 & Média & 2016 & 2017 & Média2 & 2016 & 2017 & Média & 2016 & 2017 & Média & 2016 & 2017 & Média \\
\hline Média & $4,0 \mathrm{~A}$ & $2,4 \mathrm{~B}$ & 3,2 & $5380 \mathrm{~A}$ & $755 B$ & 3067 & $0,0 \mathrm{~B}$ & $2,0 \mathrm{~A}$ & 1,0 & $2 \mathrm{~B}$ & $74 \mathrm{~A}$ & 38 & 0 & 0 & 0 \\
\hline Mín & 1,8 & 1,2 & 1,2 & 1632 & 52 & 52 & 0,0 & 0,0 & 0,0 & 0 & 0 & 0 & 0 & 0 & 0 \\
\hline Máx & 10,9 & 4,5 & 10,9 & 14833 & 1802 & 14833 & 0,0 & 22,9 & 22,9 & 18 & 354 & 354 & 0 & 0 & 0 \\
\hline $\mathrm{CV}^{1}$ & 51,4 & 36,0 & 54,3 & 61,8 & 68,8 & 108,1 & 0,0 & 250,2 & 364,6 & 212,2 & 125,9 & 195,2 & - & - & - \\
\hline $\mathrm{P}$ & & 00 & & 0,0 & & & & 001 & & 0,0 & 00 & & & - & \\
\hline
\end{tabular}

Médias seguidas de letras minúsculas distintas nas colunas diferem entre si pelo teste de Scott-Knott a 5\% de significância. Médias seguidas de letras maiúsculas distintas nas linhas indicam diferença entre os anos pelo teste $\mathrm{F}$ a $5 \%$ de significância para AV e FUM e pelo teste de Mann-Whitney a 5\% de significância para AFLA e ZEA. ${ }^{1}$ CV: coeficiente de variação (\%).

As AFLA não foram detectadas nas amostras do ano de 2016, mas em 2017 foram detectadas em $17 \%$ das amostras (Tab. 3). Essa prevalência foi similar à encontrada por Oliveira et al. (2017), que relataram uma prevalência de $19 \%$ em amostras de milho da região Sul do Brasil. As AFLA são produzidas por fungos do gênero Aspergillus e consideradas micotoxinas de grãos armazenados, pois requerem menor atividade de água do que os fungos do gênero Fusarium (Marin et al., 1998), que são produtores de FUM e ZEA, consideradas micotoxinas de campo, as quais, no presente estudo, estiveram mais prevalentes. Provavelmente neste estudo não tenha havido tempo e/ou condições adequadas após a colheita para o desenvolvimento e a produção de AFLA. As variações nas concentrações de AFLA em diferentes híbridos de milho podem estar relacionadas às concentrações do ácido linoleico e da enzima lipoxigenase (Zeringue et al., 1996). Segundo os autores, a lipoxigenação do ácido linoleico seria capaz de produzir aldeídos voláteis com cadeias de seis até 12 carbonos, inibindo ou impedindo o crescimento de fungos e a formação de AFLA. 
Tabela 4. Médias de grãos avariados (AV), fumonisinas $\left(B_{1}+B_{2}\right)(F U M)$, aflatoxinas $\left(B_{1}+B_{2}+G_{1}+G_{2}\right)$ (AFLA), zearalenona (ZEA), energia metabolizável aparente corrigida para balanço de nitrogênio (EMAn), proteína bruta (PB), aminoácidos digestíveis para aves e custo da ração inicial para frangos de corte ( $\mathrm{R} \$ / \mathrm{t}$ ração) por textura do grão, tecnologia de transgenia, tipo de híbrido e ciclo de 26 híbridos de milho cultivados nas safrinhas de 2016 e 2017

\begin{tabular}{|c|c|c|c|c|c|c|c|c|c|c|c|}
\hline Textura & $\begin{array}{l}\text { AV } \\
(\%)\end{array}$ & $\begin{array}{c}\text { FUM } \\
(\mu \mathrm{g} / \mathrm{kg})\end{array}$ & $\begin{array}{l}\text { AFLA } \\
(\mu \mathrm{g} / \mathrm{kg})\end{array}$ & $\begin{array}{c}\text { ZEA } \\
(\mu \mathrm{g} / \mathrm{kg})\end{array}$ & $\begin{array}{c}\text { EMAn } \\
\text { (kcal/kg) }\end{array}$ & $\begin{array}{l}\text { PB } \\
(\%)\end{array}$ & $\begin{array}{l}\text { LisD } \\
(\%)^{1}\end{array}$ & $\begin{array}{l}\text { MetD } \\
(\%)^{2}\end{array}$ & $\begin{array}{l}\text { CisD } \\
(\%)^{3}\end{array}$ & $\begin{array}{l}\text { TreD } \\
(\%)^{4}\end{array}$ & $\mathrm{R} \$ / \mathrm{t}$ ração \\
\hline Dentado & 2,58 & 2447 & 1,3 & 13 & 3273 & $8,37 \mathrm{ab}$ & $0,200 \mathrm{ab}$ & $0,159 \mathrm{ab}$ & $0,151 \mathrm{ab}$ & $0,286 a b$ & 673,83 \\
\hline Semidentado & 3,49 & 2940 & 0,2 & 18 & 3256 & 7,99ab & $0,194 \mathrm{~b}$ & $0,159 b$ & $0,145 b$ & $0,271 b$ & 680,28 \\
\hline Semiduro & 3,16 & 3573 & 1,9 & 50 & 3268 & $7,93 \mathrm{~b}$ & $0,192 b$ & $0,158 \mathrm{~b}$ & $0,146 \mathrm{~b}$ & $0,270 \mathrm{~b}$ & 684,35 \\
\hline Duro & 2,76 & 2615 & 0,2 & 38 & 3259 & $8,18 \mathrm{a}$ & $0,201 \mathrm{a}$ & $0,163 \mathrm{a}$ & $0,150 \mathrm{a}$ & $0,280 \mathrm{a}$ & 676,49 \\
\hline P-Textura & 0,533 & 0,436 & $0,547 *$ & $0,803 *$ & 0,276 & 0,003 & $<0,001$ & $<0,001$ & $<0,001$ & 0,001 & 0,069 \\
\hline $\begin{array}{l}\text { P-Ano } \\
\text { Tecnologia }\end{array}$ & $<0,001$ & $<0,001$ & & & $<0,001$ & 0,025 & $<0,001$ & $<0,001$ & 0,620 & $<0,001$ & $<0,001$ \\
\hline $\mathrm{HX}$ & 2,37 & 4834ab & 0,2 & 17 & 3281 & $7,91 \mathrm{~b}$ & 0,200 & $0,160 \mathrm{ab}$ & $0,148 \mathrm{ab}$ & $0,267 \mathrm{~b}$ & $685,17 \mathrm{ab}$ \\
\hline PRO & 4,68 & $2337 \mathrm{ab}$ & 0,0 & 127 & 3292 & $8,14 \mathrm{ab}$ & 0,193 & $0,159 \mathrm{ab}$ & $0,147 \mathrm{ab}$ & $0,276 a b$ & $670,28 b$ \\
\hline PRO2 & 4,67 & $7134 a$ & 0,0 & 20 & 3239 & $7,80 \mathrm{~b}$ & 0,194 & $0,154 \mathrm{~b}$ & $0,144 \mathrm{ab}$ & $0,263 b$ & $700,86 a$ \\
\hline PRO3 & 3,22 & $2970 b$ & 0,7 & 43 & 3278 & $8,05 \mathrm{ab}$ & 0,196 & $0,16 \mathrm{ab}$ & $0,148 \mathrm{ab}$ & $0,274 \mathrm{ab}$ & $677,99 b$ \\
\hline PW & 3,16 & $3177 b$ & 1,8 & 41 & 3259 & $7,94 \mathrm{~b}$ & 0,192 & $0,159 b$ & $0,145 b$ & $0,270 b$ & $683,40 \mathrm{ab}$ \\
\hline VIPTERA & 2,72 & $1737 b$ & 0,1 & 45 & 3250 & $8,27 \mathrm{a}$ & 0,200 & $0,163 \mathrm{a}$ & $0,150 \mathrm{a}$ & $0,285 \mathrm{a}$ & $674,18 b$ \\
\hline P-Tecnologia & 0,073 & 0,002 & $0,914 *$ & $0,461 *$ & $<0,001$ & 0,001 & 0,009 & $<0,001$ & 0,003 & $<0,001$ & $<0,001$ \\
\hline P-Ano & $<0,001$ & $<0,001$ & & & $<0,001$ & 0,020 & $<0,001$ & $<0,001$ & 0,575 & $<0,001$ & $<0,001$ \\
\hline \multicolumn{12}{|c|}{ Tipo de híbridı } \\
\hline Simples & 3,37 & 3358 & 0,7 & 36 & 3266 & $7,93 \mathrm{~b}$ & 0,194 & $0,159 b$ & $0,147 b$ & $0,269 \mathrm{~b}$ & 683,02 \\
\hline Simples mod. ${ }^{5}$ & 2,98 & 1905 & 0,0 & 21 & 3251 & $8,40 \mathrm{a}$ & 0,202 & $0,165 \mathrm{a}$ & $0,151 \mathrm{a}$ & $0,292 \mathrm{a}$ & 671,43 \\
\hline Duplo & 3,87 & 1864 & 0,0 & 82 & 3265 & $8,48 \mathrm{a}$ & 0,204 & $0,166 \mathrm{a}$ & $0,149 \mathrm{ab}$ & $0,289 \mathrm{a}$ & 667,67 \\
\hline Triplo & 2,67 & 3286 & 2,3 & 42 & 3261 & $8,01 \mathrm{~b}$ & 0,193 & $0,159 b$ & $0,146 b$ & $0,273 b$ & 682,18 \\
\hline P-Tipo & 0,239 & 0,372 & $0,599 *$ & $0,433 *$ & 0,286 & $<0,001$ & 0,007 & $<0,001$ & 0,022 & $<0,001$ & 0,013 \\
\hline P-Ano & $<0,001$ & $<0,001$ & & & $<0,001$ & 0,017 & $<0,001$ & $<0,001$ & 0,544 & 0,001 & $<0,001$ \\
\hline \multicolumn{12}{|l|}{ Ciclo } \\
\hline Superprecoce & $3,04 \mathrm{~b}$ & $4431 \mathrm{a}$ & 0,6 & 43 & 3268 & $7,95 b$ & $0,194 \mathrm{a}$ & $0,158 b$ & $0,147 \mathrm{a}$ & $0,269 \mathrm{~b}$ & $686,33 \mathrm{~b}$ \\
\hline Precoce & $2,98 b$ & $2053 b$ & 1,2 & 27 & 3260 & $8,09 a$ & $0,196 \mathrm{a}$ & $0,161 \mathrm{a}$ & $0,148 \mathrm{a}$ & $0,277 \mathrm{a}$ & $675,91 \mathrm{c}$ \\
\hline Normal & $7,20 \mathrm{a}$ & $5091 \mathrm{a}$ & 5,9 & 133 & 3264 & $7,45 \mathrm{c}$ & $0,178 b$ & $0,153 \mathrm{c}$ & $0,138 b$ & $0,248 \mathrm{c}$ & $704,66 \mathrm{a}$ \\
\hline P-Ciclo & $<0,001$ & $<0,001$ & $0,051 *$ & $0,446^{*}$ & 0,122 & $<0,001$ & $<0,001$ & $<0,001$ & $<0,001$ & $<0,001$ & $<0,001$ \\
\hline P-Ano & $<0,001$ & $<0,001$ & & & 0,000 & 0,019 & $<0,001$ & $<0,001$ & 0,538 & 0,001 & $<0,001$ \\
\hline $\mathrm{CV}^{6}(\%)$ & 68,6 & 122,9 & 661,0 & 261,6 & 1,7 & 4,8 & 6,5 & 3,6 & 4,0 & 6,0 & 3,9 \\
\hline
\end{tabular}

Médias seguidas de letras distintas nas colunas diferem entre si pelo teste de Tukey a 5\% de significância. ${ }^{1}$ LisD: lisina digestível para aves. ${ }^{2}$ MetD: metionina digestível para aves. ${ }^{3} \mathrm{CisD}$ : cistina digestível para aves. ${ }^{4} \mathrm{TreD}$ : treonina digestível para aves. *Valor do P para o teste de Kruskal-Wallis. ${ }^{5}$ Simples mod.: simples modificado. ${ }^{6} \mathrm{CV}$ : coeficiente de variação.

Em 2016, somente $8 \%$ das amostras apresentaram contaminações por ZEA, enquanto em 2017, essa toxina foi detectada em $59 \%$ das amostras. Houve diferença de concentração em apenas cinco híbridos nos dois anos $(\mathrm{P}<0,05)$, tendo a maior concentração média $(\mathrm{P}<0,05)$ ocorrido na safrinha de 2017 (Tab. 3). Essas variações são descritas na literatura, em Oliveira et al. (2017), que diagnosticaram prevalência de ZEA de $74 \%$, e em De Souza et al. (2013), que verificaram apenas $12 \%$ de prevalência dessa micotoxina.

Não foi detectado DON $(\geq 200 \mu \mathrm{g} / \mathrm{kg})$ nas amostras de milhos cultivados nos dois anos. Esses dados diferenciam-se da prevalência de $33 \%$ constatada por Oliveira et al. (2017), porém estão mais próximos dos $4 \%$ encontrados por Souza et al. (2013) ao avaliarem 74 amostras de milho coletadas de uma integradora de frangos localizada no Paraná. Grãos que sofreram danos por fungos, fragmentos de grãos e impurezas podem ter maior concentração de micotoxinas que grãos aparentemente sadios (Shetty e Bhat, 1999). No entanto, no presente estudo, observouse fraca correlação linear no ano de 2017 entre grãos avariados e FUM $\left(\mathrm{P} \leq 0,028\right.$ e $\left.\mathrm{R}^{2}=0,06\right)$, bem como entre grãos avariados e ZEA $(\mathrm{P}<0,001$ e $\left.\mathrm{R}^{2}=0,16\right)$, e moderada correlação de postos de Spearman entre grãos avariados e AFLA $(\mathrm{P}<0,001$ e $\mathrm{R}=0,42)$. 
Não houve agrupamento dos híbridos para EMAn no ano de 2016 e, em 2017, os híbridos distribuíram-se em três grupos distintos $(\mathrm{P}<0,05)$. Dos 26 híbridos avaliados, 21 tiveram média de EMAn maior $(\mathrm{P}<0,05)$ na safrinha do ano de 2017 em relação ao de 2016. A EMAn média nos dois anos foi de $3264 \mathrm{kcal} / \mathrm{kg}$, apresentando interação $(\mathrm{P}<0,05)$ entre os anos
(Tab. 5). Rostagno et al. (2011) apontam o valor médio de $3.381 \mathrm{kcal} / \mathrm{kg}$ para o milho, o qual foi semelhante ao grupo de híbridos com maior EMAn no ano de 2017 no presente estudo. Vieira et al. (2007), ao avaliarem 45 híbridos, constataram EMAn média de $3251 \mathrm{kcal} / \mathrm{kg}$, muito semelhante ao resultado deste estudo, porém com maior amplitude de variação $(506 \mathrm{kcal} / \mathrm{kg})$.

Tabela 5. Médias de energia metabolizável aparente corrigida para balanço de nitrogênio (EMAn), proteína bruta $(\mathrm{PB})$, aminoácidos digestíveis para aves e custo da ração inicial para frangos de corte $(\mathrm{R} \$ / \mathrm{t}$ ração) de 26 híbridos de milho cultivados nas safrinhas de 2016 e 2017

\begin{tabular}{|c|c|c|c|c|c|c|c|c|c|c|c|c|c|}
\hline \multirow{2}{*}{\multicolumn{3}{|c|}{$\mathrm{H}^{1}$ EMAn $(\mathrm{kcal} / \mathrm{kg})$}} & \multicolumn{2}{|c|}{$\mathrm{PB}(\%)$} & \multicolumn{2}{|c|}{$\operatorname{LisD}^{2}(\%)$} & \multicolumn{2}{|c|}{$\operatorname{MetD}^{3}(\%)$} & \multicolumn{2}{|c|}{$\operatorname{CisD}^{4}(\%)$} & \multicolumn{2}{|c|}{$\operatorname{TreD}^{5}(\%)$} & Ração (R\$/t) \\
\hline & & 2017 & 2016 & 2017 & 2016 & 2017 & 2016 & 2017 & 2016 & 2017 & 2016 & 2017 & $2016 \quad 2017$ \\
\hline 1 & $3236 \mathrm{~A}$ & $3317 \mathrm{bB}$ & & & $0,191 \mathrm{~b} A$ & $0,180 \mathrm{bB}$ & ), $151 \mathrm{bF}$ & $15 / 0$ & $0,141 b$ & $0,145 b$ & $0,261 \mathrm{c}$ & 0,25417 & $, 13 \mathrm{aA} 67,13 \mathrm{bB}$ \\
\hline 2 & $3227 \mathrm{~B}$ & 3313bA & & & & & & $0,159 \mathrm{bB}$ & $0,143 b$ & 0,14 & $0,284 a$ & & $03,00 \mathrm{aA} 63,11 \mathrm{bB}$ \\
\hline 3 & $3235 B$ & $3263 \mathrm{cA}$ & $7,73 \mathrm{bF}$ & $3,08 \mathrm{~b}$ & $0,194 b$ & $0,193 \mathrm{a}$ & $0,157 \mathrm{~b}$ & $0,160 \mathrm{~b}$ & $0,140 \mathrm{bF}$ & 0,15 & 0,271 & $0,2 /$ & $88,61 \mathrm{bA} 66,96 \mathrm{bB}$ \\
\hline 4 & 3213B & $3313 b A$ & $3,14 \mathrm{aF}$ & $3,46 \mathrm{aA}$ & $0,200 \mathrm{~b}$ & $0,193 \mathrm{a}$ & $0,160 \mathrm{a}$ & 0,159 & $0,140 \mathrm{bF}$ & 0,150 & $0,284 a$ & $0,276: 6$ & $93,72 \mathrm{bA} 51,90 \mathrm{cB}$ \\
\hline 5 & 3168B & $3292 \mathrm{cA}$ & 7,99a & $8,20 \mathrm{~b}$ & $0,207 \mathrm{a}$ & $0,189 a$ & J,153bF & $0,160 \mathrm{bA}$ & $0,148 \mathrm{a}$ & 0,1 & $0,273 b$ & $0,273: 6$ & $98,61 \mathrm{bA} 61,68 \mathrm{bB}$ \\
\hline 6 & 3219B & $3296 \mathrm{cA}$ & $7,96 \mathrm{~b}$ & $8,32 \mathrm{~b}$ & $0,201 b$ & $0,194 a$ & $0,161 \mathrm{a}$ & $0,167 \mathrm{a}$ & $0,147 \mathrm{a}$ & 0,1 & $0,281 \mathrm{a}$ & $0,281: 6$ & $86,97 \mathrm{bA} 55,64 \mathrm{cB}$ \\
\hline 7 & 3230 & $3297 \mathrm{c}$ & $7,42 \mathrm{c}$ & $7,49 \mathrm{~d}$ & $0,180 \mathrm{~b}$ & $0,176 b$ & $0,15 b$ & $0,156 \mathrm{~b}$ & $0,137 b$ & 0,1 & $0,244 \mathrm{c}$ & 0,25317 & $26,05 \mathrm{aA}, 83,26 \mathrm{aB}$ \\
\hline 8 & 3215 & $3316 b$ & $8,14 a$ & $8,64 \mathrm{a}$ & $0,206 \mathrm{a}$ & $0,196 \mathrm{a}$ & $0,155 b$ & $0,166 \mathrm{a}$ & $0,149 \mathrm{a}$ & & $0,281 \mathrm{a}$ & $0,285 i$ & $692,44 \mathrm{~b} 648,48 \mathrm{c}$ \\
\hline 9 & 3264 & $3321 b$ & $7,93 b$ & $7,49 \mathrm{~d}$ & $0,193 b$ & $0,176 \mathrm{~b}$ & $0,160 \mathrm{a}$ & $0,160 \mathrm{~b}$ & $0,140 b$ & & $0,270 b$ & 0,24 & $77,55 \mathrm{bA} 64,66 \mathrm{bB}$ \\
\hline 10 & $3216 \mathrm{~B}$ & $3350 \mathrm{aA}$ & $7,81 \mathrm{~b}$ & $7,75 \mathrm{c}$ & $0,212 \mathrm{Aa}$ & $0,179 \mathrm{bB}$ & 0,157 & $0,163 \mathrm{a}$ & $0,157 \mathrm{a}$ & 0,1 & $0,277 \mathrm{a}$ & 0,25217 & $21,26 \mathrm{aA} 58,65 \mathrm{bB}$ \\
\hline 11 & $3235 \mathrm{~B}$ & 3333 & & $8,32 \mathrm{~b}$ & $0,205 \mathrm{a}$ & $0,193 \mathrm{a}$ & 0,154 & $0,163 \mathrm{a}$ & $0,147 \mathrm{a}$ & 0,1 & 0,276 & 0,21 & $96 \mathrm{cB}$ \\
\hline 12 & $3204 \mathrm{~B}$ & $3272 \mathrm{c}$ &, $12 \mathrm{a}$ & $48 \mathrm{a}$ & $0,201 \mathrm{bA}$ & $0,190 \mathrm{aB}$ & 0,157 & $0,160 \mathrm{~b}$ & $0,147 \mathrm{a}$ & 0,1 & 0,281 & 0,28 & $16,58 \mathrm{aA} 59,15 \mathrm{bB}$ \\
\hline 13 & $3235 \mathrm{~B}$ & $3287 \mathrm{c} A$ & $7,91 \mathrm{~b}$ & $8,10 \mathrm{~b}$ & $0,198 b$ & $0,192 \mathrm{a}$ & $0,160 a$ & $0,165 \mathrm{a}$ & $0,145 b$ & 0,1 & 0,278 & 0,27 & $33 \mathrm{bB}$ \\
\hline 14 & 3223 & $3312 b$ & $7,60 \mathrm{c}$ & $7,79 \mathrm{c}$ & $0,194 b$ & $0,183 b$ & 0,15 & $0,159 \mathrm{~b}$ & $0,146 \mathrm{a}$ & 0,1 & 0,265 & 0,2 & ,96b \\
\hline 15 & $3205 B$ & 3319 & $7,90 \mathrm{~b}$ & $8,20 \mathrm{~b}$ & $0,203 a$ & 0,1 & 0,1 & $162 b$ & $0,145 b$ & 0,1 & 0,273 & & $06 \mathrm{~b}$ \\
\hline 16 & 3203B & 328 & $8,47 \mathrm{a}$ & $8,63 \mathrm{a}$ & $0,213 \mathrm{a}$ & $0,197 \mathrm{a}$ & 0,16 & $167 \mathrm{a}$ & $0,152 \mathrm{a}$ & 0,1 & 0,301 & $\sigma$ & $05 \mathrm{cB}$ \\
\hline 17 & 3198B & 329 & $7,64 \mathrm{c}$ & $8,00 \mathrm{~b}$ & $0,200 \mathrm{~b}$ & $0,186 a$ & 0,1 & $156 \mathrm{~b}$ & $0,144 b$ & 0,1 & 0,26 & 0,2 & $64 \mathrm{bB}$ \\
\hline 18 & 3203B & $3320 \mathrm{~A}$ & $8,23 \mathrm{a}$ & $8,46 \mathrm{a}$ & $0,209 \mathrm{a}$ & 0,1 & 0,1 & $0,164 \mathrm{a}$ & $0,153 \mathrm{a}$ & & 0,29 & 0,2 & $4 \mathrm{cB}$ \\
\hline 19 & $3227 \mathrm{~B}$ & 330 & $8,35 \mathrm{a}$ & $8,59 a$ & $0,212 \mathrm{a}$ & $0,196 \mathrm{a}$ & 0,16 & $0,169 \mathrm{aA}$ & $0,148 \mathrm{a}$ & 0,1 & 0,2 & 0,2 & $9 \mathrm{cB}$ \\
\hline 20 & $3196 \mathrm{~B}$ & & $8,07 \mathrm{a}$ & $7,88 \mathrm{c}$ & $0,197 b$ & $0,187 \mathrm{a}$ & 0,1 & $0,160 \mathrm{~b}$ & $0,149 \mathrm{a}$ & 0,1 & $0,2 / 8 \mathrm{a} A$ &, 26 & $62 \mathrm{bB}$ \\
\hline 21 & $3223 \mathrm{~A}$ & 334 & $7,38 \mathrm{c}$ & $7,23 \mathrm{~d}$ & $0,192 \mathrm{bA}$ & $0,173 \mathrm{bB}$ & 0,1 & $0,160 \mathrm{~b}$ & $0,141 b$ & 0,1 & 0,25 & 0,2 & $4 \mathrm{bB}$ \\
\hline 22 & 3202 & $3318 b$ & $8,33 \mathrm{a}$ & $8,18 \mathrm{~b}$ & $0,214 \mathrm{a}$ & $0,183 b$ & 0,16 & $0,166 \mathrm{a}$ & $0,153 \mathrm{a}$ & 0,15 & $0,292 \mathrm{a}$ & 0,2 & ,47c \\
\hline 23 & 3233 & $3351 \mathrm{a}$ & $8,19 \mathrm{a}$ & $8,08 \mathrm{~b}$ & $0,203 a$ & $0,183 b$ & $0,159 \mathrm{a}$ & $0,159 b$ & $0,149 \mathrm{a}$ & $0,146 \mathrm{a}$ & $0,284 \mathrm{a}$ & $0,269: 6$ & $92,24 \mathrm{bA} 448,33 \mathrm{cB}$ \\
\hline 24 & 3228 & $3318 b$ & $7,94 b$ & $8,11 \mathrm{~b}$ & $0,200 \mathrm{~b}$ & $0,190 \mathrm{a}$ & $0,154 b$ & $0,160 \mathrm{~b}$ & $0,147 \mathrm{a}$ & $0,146 \mathrm{a}$ & $0,273 b$ & 0,266 & $03,26 \mathrm{aA} 58,46 \mathrm{bB}$ \\
\hline 25 & 3235 & $3361 \mathrm{a}$ & $7,76 b$ & $7,80 \mathrm{c}$ & $0,214 \mathrm{a}$ & $0,196 \mathrm{a}$ & $0,163 \mathrm{a}$ & $0,166 \mathrm{a}$ & $0,149 \mathrm{a}$ & $0,150 \mathrm{a}$ & $0,271 b$ & 0,25617 & $18,23 \mathrm{aA} i 54,72 \mathrm{cB}$ \\
\hline 26 & 3174 & $3308 b$ & $8,08 \mathrm{a}$ & $8,16 b$ & $0,208 \mathrm{a}$ & $0,192 \mathrm{a}$ & $0,163 \mathrm{a}$ & $0,167 \mathrm{a}$ & $0,156 \mathrm{a}$ & $0,147 \mathrm{a}$ & $0,292 \mathrm{a}$ & $0,269: 6$ & $91,22 \mathrm{bA} 56,57 \mathrm{cB}$ \\
\hline $\mathrm{P}$ & 0,241 & $<0,001$ & 0,000 & 0,000 & 0,041 & $<0,001$ & $<0,001$ & 0,002 & $<0,006$ & $<0,003$ & 0,000 & 0,000 & $0,000<0,001$ \\
\hline Méd & $3217 \mathrm{~B}$ & $3312 \mathrm{~A}$ & $7,95 \mathrm{~B}$ & $8,08 \mathrm{~A}$ & $0,202 \mathrm{~A}$ & $0,188 \mathrm{~B}$ & $0,157 \mathrm{~B}$ & $0,162 \mathrm{~A}$ & 0,147 & 0,147 & $0,277 \mathrm{~A}$ & $0,269 I^{\prime}$ & $702,36 \mathrm{~A} 558,86 \mathrm{~B}$ \\
\hline Mín & 3168 & 3263 & 7,38 & 7,23 & 0,180 & 0,173 & 0,150 & 0,156 & 0,137 & 0,14 & 0,244 & 0,243 & $677,55 \quad 645,96$ \\
\hline Máx & 3264 & 3361 & 8,47 & 8,64 & 0,214 & 0,197 & 0,163 & 0,169 & 0,157 & 0,151 & 0,301 & 0,301 & $728,77 \quad 683,26$ \\
\hline $\mathrm{CV}^{6}$ & 0,6 & 0,7 & 3,4 & 4,6 & 4,1 & 3,7 & 2,5 & 2,3 & 3,5 & 2,3 & 4,4 & 5,2 & $2,0 \quad 1,2$ \\
\hline $\mathrm{P}$ & & & & & & & 0,0 & 000 & & & 0,0 & & 0,000 \\
\hline
\end{tabular}

Médias seguidas de letras minúsculas distintas nas colunas diferem entre si pelo teste de Scott-Knott a $5 \%$ de significância. Médias seguidas de letras maiúsculas distintas nas linhas indicam diferença entre os anos pelo teste $\mathrm{F}$ a 5\% de significância. ${ }^{1} \mathrm{H}$ : híbrido. ${ }^{2}$ LisD: lisina digestível para aves. ${ }^{3} \mathrm{MetD}$ : metionina digestível para aves. ${ }^{4}$ CisD: cistina digestível para aves. ${ }^{5}$ TreD: treonina digestível para aves. ${ }^{6} \mathrm{CV}$ : coeficiente de variação (\%).

Os resultados de PB dos híbridos de milho distribuíram-se em três grupos em 2016 e quatro grupos em 2017 (Tab. 5). Somente três híbridos tiveram média de $\mathrm{PB}$ maior $(\mathrm{P}<0,05)$ na safrinha do ano de 2017 em relação ao de 2016. Contudo, a média de concentração do ano de 2017 foi maior $(\mathrm{P}<0,05)$. Valor médio superior $(8,61 \%)$ foi encontrado por Piovesan et al. (2010) em trabalho com 45 amostras de grãos de milho na região do oeste do Paraná. Valores inferiores $(7,17 \%)$ e variação maior $(5,82-8,80 \%)$ foram reportados por Vieira et al. (2007) em estudo 
com 45 híbridos, demonstrando que a PB sofre influência de híbrido, safra e região de cultivo. No presente estudo, a PB teve fraca correlação negativa com EMAn $\left(\mathrm{R}^{2}=0,15\right.$ e $\left.\mathrm{P}<0,001\right)$ em 2016 e também em 2017.

Os níveis de LisD, MetD, CisD e TreD nos diferentes híbridos distribuíram-se em dois grupos nas safrinhas de 2016 e 2017, com exceção da TreD, que, em 2016, distribuiu-se em três grupos (Tab. 5). Na média geral por ano, percebeu-se que LisD e TreD foram maiores $(\mathrm{P}<0,05)$ em 2016. MetD teve maior $(\mathrm{P}<0,05)$ concentração em 2017 e CisD não teve efeito de ano de cultivo.

Verificou-se que híbridos com grãos duros tiveram maior $(\mathrm{P}<0,05) \quad \mathrm{PB}$ e aminoácidos digestíveis (Tab. 4). Dos cinco híbridos com textura dura, quatro são da tecnologia de transgenia Viptera, os quais tiveram maiores
$(\mathrm{P}<0,05)$ concentrações de $\mathrm{PB}$, MetD, CisD, TreD e menor custo da ração $(\mathrm{P}<0,05)$. Quanto ao tipo, constatou-se que híbridos simples modificados e duplos tiveram maiores $(\mathrm{P}<0,05)$ percentuais de PB, MetD, CisD e TreD. Quanto ao ciclo, o precoce teve maiores $(\mathrm{P}<0,05)$ percentuais de PB, LisD, MetD, CisD, TreD e menor $(\mathrm{P}<0,05)$ custo da ração.

$\mathrm{O}$ custo da ração foi maior $(\mathrm{P}<0,05)$ no ano de 2016, quando os híbridos se distribuíram em dois grupos. Em 2017 houve três grupos, e a média de custo dos dois anos foi de $\mathrm{R} \$ 680,60$ por tonelada (Tab. 5). O custo da ração ajustou-se aos modelos de regressão linear múltipla, nos quais as variáveis FUM, EMAn e PB apresentaram interação $(\mathrm{P}<0,05)$ no ano de 2016 , bem como na avaliação conjunta dos dois anos, e FUM, EMAn, PB e grãos avariados apresentaram interação em 2017 (Tab. 6).

Tabela 6. Modelos de regressão linear múltipla para o custo da ração inicial para frangos de corte (R $\$ / t$ ração) calculado com 26 híbridos de milho cultivados nas safrinhas de 2016 e 2017

\begin{tabular}{|c|c|c|c|c|c|c|}
\hline \multirow[b]{2}{*}{ Ano } & \multirow[b]{2}{*}{ Equação } & \multirow{2}{*}{$\begin{array}{c}\mathrm{R}^{2} \\
\text { ajustado }\end{array}$} & \multicolumn{4}{|c|}{$\%$ do $\mathrm{R}^{2}$ ajustado } \\
\hline & & & EMAn $^{1}$ & $\mathrm{~PB}^{2}$ & FUM & $\mathrm{AV}^{4}$ \\
\hline 2016 & Custo $=1651,83-17,12 *$ PB-0,26*EMAn+0,0029*FUM & 0,76 & 18,9 & 9,7 & 71,4 & $\mathrm{NS}^{5}$ \\
\hline 2017 & $\begin{array}{c}\text { Custo }=1634,85-17,34 * \mathrm{~PB}- \\
0,25 * \mathrm{EMAn}+0,0031 * \mathrm{FUM}+1,12 * \mathrm{AV}\end{array}$ & 0,74 & 61,0 & 29,4 & 7,9 & 1,7 \\
\hline $\begin{array}{l}2016 \text { e } \\
2017\end{array}$ & Custo $=1729,68-18,35 *$ PB- $0,28 *$ EMAn $+0,0030 *$ FUM & 0,92 & 69,5 & 24,2 & 6,3 & NS \\
\hline
\end{tabular}

${ }^{1}$ EMAn: energia metabolizável aparente corrigida para balanço de nitrogênio. ${ }^{2} \mathrm{~PB}$ : proteína bruta. ${ }^{3} \mathrm{FUM}$ : fumonisinas $\left(\mathrm{B}_{1}+\mathrm{B}_{2}\right) .{ }^{4} \mathrm{AV}$ : grãos avariados. ${ }^{5} \mathrm{NS}$ : não significativo $(\mathrm{P}>0,05)$

As dietas comumente utilizadas na avicultura de corte têm o milho como principal ingrediente e fonte de energia (Moreira et al., 1994), porém constatou-se, no presente estudo, que, além da EMAn, as FUM e a PB também tiveram efeito significativo no custo da ração, inclusive com variações de uma safra para outra. Em 2016, o custo da ração foi fortemente influenciado pela concentração de FUM, pois quanto maior a concentração no milho, maior a inclusão de AAM e, consequentemente, maior o custo da ração. No entanto, em 2017, quando a concentração por FUM foi menor, a EMAn teve maior influência no modelo.

Realizou-se um PCA para cada ano, com as variáveis FUM, grãos avariados, EMAn, PB,
LisD, MetD, TreD, CisD e custo da ração, em que dois componentes principais (PCs) foram observados: PC1 e PC2 (Fig. 1). Em 2016, o PC1 e o PC2 contribuíram com $50 \%$ e $21 \%$, respectivamente, o que explica $71 \%$ da variação total dos dados. Em 2017, o PC1 e o PC2 contribuíram com $57 \%$ e $17 \%$, respectivamente, o que esclarece $74 \%$ da variação total. Nos dois anos de experimento, houve cinco híbridos com textura dura, dentre os quais quatro, com tecnologia Viptera, formaram um grupo que se correlacionou negativamente ao custo da ração e de FUM. Além disso, é possível observar que quanto menor o percentual de PB e aminoácidos digestíveis, maior é o custo da ração e maior é a concentração de FUM. 


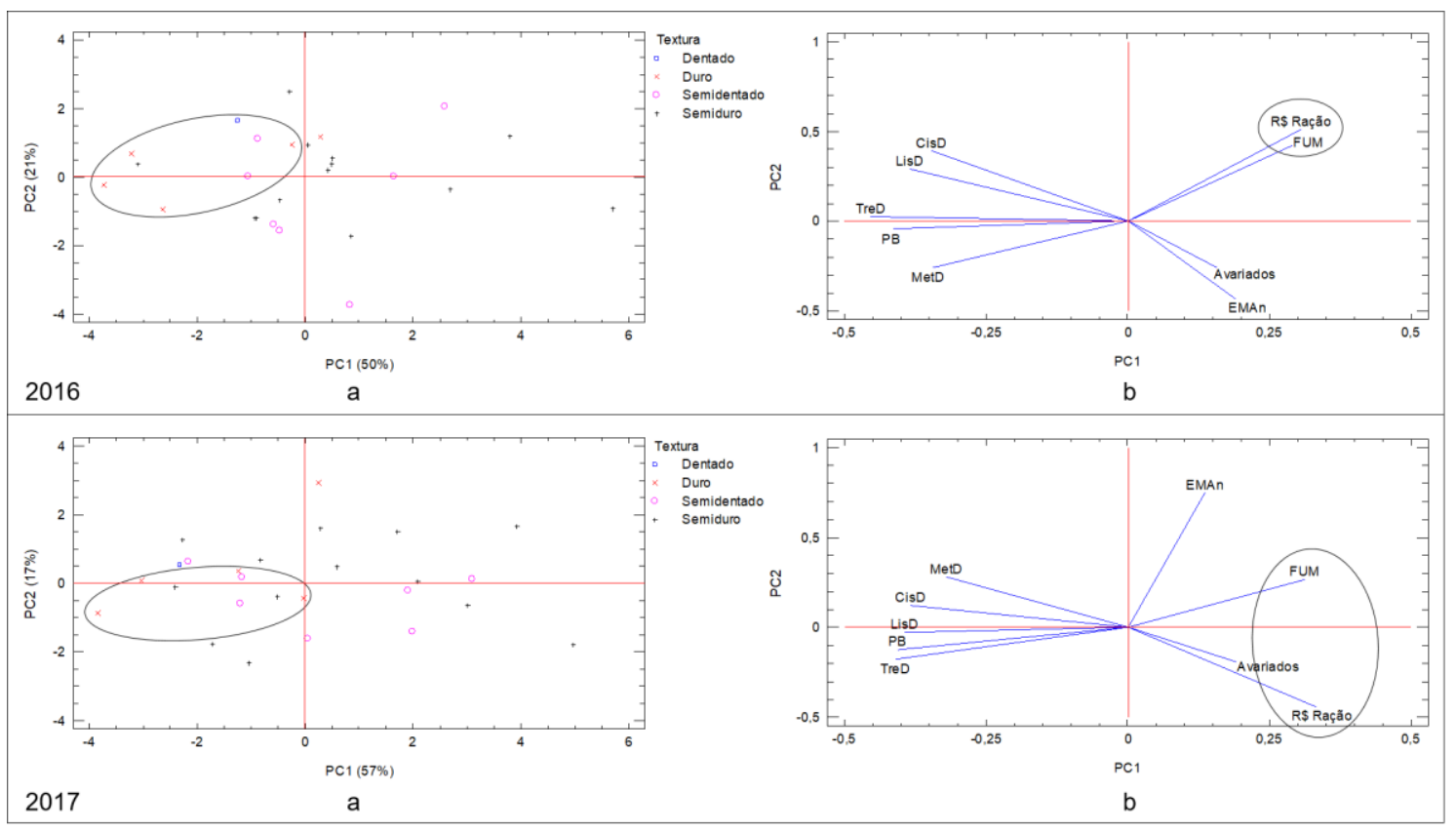

Figura 1. Gráfico dos scores (a), por tipo de textura de grão, e loadings (b) para amostras de 26 híbridos de milho cultivados nas safrinhas de 2016 e 2017 analisadas para PCA. Correlação entre grãos avariados, fumonisinas $\left(\mathrm{B}_{1}+\mathrm{B}_{2}\right)(\mathrm{FUM})$, energia metabolizável aparente corrigida para balanço de nitrogênio (EMAn), proteína bruta (PB), aminoácidos digestíveis para aves (LisD, MetD, CisD e TreD) e custo da ração inicial para frangos de corte ( $\mathrm{R} \$$ ração).

Mesmo que não tenha havido efeito significativo dos aminoácidos digestíveis sobre o custo da ração no modelo de regressão linear múltipla, na PCA é possível visualizar correlação positiva entre PB e aminoácidos digestíveis e correlação negativa entre $\mathrm{PB}+$ aminoácidos digestíveis e custo da ração+FUM (Fig. 1).

\section{CONCLUSÃO}

A composição micotoxicológica e nutricional é influenciada pelos diferentes híbridos e também pelo ano de cultivo e, por isso, deve ser constantemente monitorada para a produção de rações. A correlação entre grãos avariados e micotoxinas é de fraca magnitude, evidenciando a necessidade de análises laboratoriais para quantificação da concentração de micotoxinas. Altas concentrações de micotoxinas influenciam negativamente no custo da ração, pois requerem a inclusão de aditivos antimicotoxinas. Híbridos com tecnologia Viptera apresentam menor concentração de FUM, maiores percentuais de PB, MetD, CisD e TreD e, consequentemente, produzem ração de custo mais baixo. Híbridos simples modificados e duplos têm maiores percentuais de $\mathrm{PB}$, MetD, CisD e TreD, e híbridos de ciclo precoce têm menor concentração de FUM, maiores percentuais de $\mathrm{PB}$, LisD, MetD, CisD, TreD e produzem ração de menor custo.

\section{REFERÊNCIAS}

BERTHILLER, F.; SCHUHMACHER, R.; BUTTINGER, G.; KRSKA, R. Rapid simultaneous determination of major type A- and B-trichothecenes as well as zearalenone in maize by high performance liquid chromatography-tandem mass spectrometry. $J$. Chromatogr. A, v.1062, p.209-216, 2005.

BRASIL. Ministério da Agricultura, Pecuária e Abastecimento. Instrução Normativa ${ }^{\circ} 60$, de 22 de dezembro de 2011. Estabelece o regulamento técnico do milho. Diário Oficial da União. Brasília, 23 dez. 2011. Seção 1, p.3-5.

BUERSTMAYR, H.; STEINER, B.; HARTL, L. et al. Molecular mapping of QTLs for Fusarium head blight resistance in spring wheat. II. Resitance to fungal penetration and spread. Theor. Appl. Genet., v.107, p.503-508, 2003.

BÚTRON, A.; REVILLA, P.; ORDÁS, A.; MALVAR, R.A. Resistance to reduce corn borer damage in maize for bread, in Spain. Crop Prot., v.28, p.1-5, 2009. 
CAMARGOS, S.M.; SOARES, L.M.V.; SAWAZAKI, E. Fumonisins in corn cultivars in the state of São Paulo. Braz. J. Microbiol., v.31, p.226-229, 2000.

DILKIN, P.; MALLMANN， C.A.; ALMEIDA, C.A.A. et al. Production of fumonisins by strains of Fusarium moniliforme according to temperature, moisture and growth period. Braz. J. Microbiol., v.33, p.111-118, 2002.

EFEITOS da gangorra agropecuária. Boletim informativo do setor, maio/2017. Disponível em: $<$ http://sindiracoes.org.br/produtos-e-servicos/boletiminformativo-do-setor/>. Acessado em: 02 jan. 2018.

EYNG, C.; NUNES, R.V.; POZZA, P.C. et al. Composição química e valores energéticos de cultivares de milho para aves. Rev. Bras. Saúde Prod. Anim., v.10, p.60-72, 2009.

HAMMOND, B.G.; CAMPBELL, K.W.; PILCHER, C.D. et al. Lower fumonisin mycotoxin levels in the grain of Bt corn grow in the United States in 20002002. J. Agric. Food Chem., v.52, p.1390-1397, 2004.

MALLMANN, A.O.; MARCHIORO, A.; OLIVEIRA, M.S. et al. Two sampling plans for fumonisins analysis in maize. Cienc. Rural, v.43, p.551-558, 2013.

MARIN, S.; SANCHIS, V.; RAMOS, A.J. et al. Environmental factors, in vitro interactions, and niche overlap between Fusarium moniliforme, $F$. proliferatum, and $F$. graminearum, Aspergillus and Penicillium species from maize grain. Mycol. Res., v.102, p.831-837, 1998.

MOREIRA, I.; ROSTAGNO, H.S.; COELHO, D.T. et al. Determinação dos coeficientes de digestibilidade, valores energéticos e índices de controle de qualidade do milho e soja integral processados a calor. Rev. Soc. Bras. Zootec., v.23, p.916-929, 1994.

MURUGESAN, G.R.; LEDOUX, D.R.; NAEHRER, K. et al. Prevalence and effects of mycotoxins on poultry health and performance, and recent development in mycotoxin counteracting strategies. Poult. Sci., v.94, p.1298-1315, 2015.

OLIVEIRA, M.S.; DIEL, A.C.L.; RAUBER, R.H. et al. Free and hidden fumonisins in Brazilian raw maize samples. Food Control, v.53, p.217-221, 2015.

OLIVEIRA, M.S.; ROCHA, A.; SULYOK, M. et al. Natural mycotoxin contamination of maize (Zea mays L.) in the South region of Brazil. Food Control, v.73, Part B, p.127-132, 2017.

OLIVEIRA, T.R.; JACCOUD-FILHO, D.S.; HENNEBERG, L. Maize (Zea mays L) landraces from the southern region of Brazil: contamination by Fusarium sp., zearalenone, physical and mechanical characteristics of the kernels. Braz. Arch. Biol. Technol., v.52, p.1-5, 2009.
ONO, E.Y.S.; BIAZON, L.; SILVA, M. et al. Fumonisins in corn: correlation with Fusarium sp. count, damaged kernels, protein and lipid content. Braz. Arch. Biol. Technol., v.49, p.63-71, 2006.

PINTO, N.F.J.A; VARGAS, E.A.; PREIS, R.A. Qualidade sanitária e produção de fumonisina $B_{1} \mathrm{em}$ grãos de milho na fase de pré-colheita. Summa Phytopathol., v.33, p.304-306, 2007.

PIOVESAN, V.; OLIVEIRA, V.; ARAÚJO, J.S. Predição do conteúdo de aminoácidos essenciais do grão de milho. Ciênc. Agrotec., v.34, p.758-764, 2010.

PNE: precise nutrition evaluation service. Antony, France: Disseo France S.A.S., 2017. Disponível em: <https://pne.adisseo.com>. Acessado em: 25 jun. 2017.

ROSTAGNO, H.S.; ALBINO, L.F.T.; DONZELE, J.L. et al. Tabelas brasileiras para aves e suínos: composição de alimentos e exigências nutricionais. 3.ed. Viçosa: Universidade Federal de Viçosa, Departamento de Zootecnia, 2011. 252p.

SHETTY, P.F.; BHAT, R.V. A physical method for segregation of fumonisin contaminated maize. Food Chem., v.66, p.371-374, 1999.

SILVA, C.S.; COUTO, H.P.; FERREIRA, R.A. et al. Valores nutricionais de milhos de diferentes qualidades para frangos de corte. Rev. Bras. Zootec., v.37, p.883-889, 2008.

SOUZA, M.D.L.M.; SULYOK, M.; FREITASSILVA, O. et al. Cooccurrence of mycotoxins in maize and poultry feeds from Brazil by liquid chromatography/tandem mass spectrometry. Sci. World J., v.2013, p.1-9, 2013.

SULYOK, M.; BERTHILLER, F.; KRSKA, R.; SCHUHMACHER, R. A liquid chromatography/tandem mass spectrometric multimycotoxin method for the quantification of 87 analytes and its application to semi-quantitative screening of moldy food samples. Anal. Bioanal. Chem., v.389, p.1505-1523, 2007.

VIEIRA, R.O.; RODRIGUES, P.B.; FREITAS, R.T.F. et al. Composição química e energia metabolizável de híbridos de milho para frangos de corte. Rev. Bras. Zootec., v.36, p.832-838, 2007.

WORLD agricultural supply and demand estimates, WASDE-572. USDA, 2017. Available in: <https://www.usda.gov/oce/commodity/wasde/latest.p df>. Accessed in: 02 Jan. 2018.

ZERINGUE, H.J.; BROWN, R.L.; NEUCERE, J.N. et al. Relationships between C6-C12 alkanal and alkenal volatile contents and resistance of maize genotypes to Aspergillus flavus and aflatoxin prodution. J. Agric. Food Chem., v.44, p.403-407, 1996. 